\title{
Prediction of Displacements in Unstable Areas Using a Neural Model
}

\author{
E. BINAGHI ${ }^{1}$, M. BOSCHETTI ${ }^{2}$, P. A. BRIVIO ${ }^{2}$, I. GALLO ${ }^{1}$, F. PERGALANI $^{3 \star}$ \\ and A. RAMPINI ${ }^{1}$ \\ ${ }^{1}$ Istituto per le Tecnologie Informatiche Multimediali, Consiglio Nazionale delle Ricerche, via \\ Ampère 56, 20131 Milan, Italy; ${ }^{2}$ Istituto per il Rilevamento Elettromagnetico dell'Ambiente, \\ Consiglio Nazionale delle Ricerche, via Bassini 15, 20133 Milan, Italy; ${ }^{3}$ Dipartimento di \\ Ingegneria Strutturale, Politecnico di Milano, p.le Leonardo da Vinci 32, 20133 Milan, Italy; \\ ^Author for correspondence (e-mail:pergalani@stru.polimi.it)
}

(Received 29 December 2000; accepted 6 June 2003)

\begin{abstract}
In pipeline management the accurate prediction of weak displacements is a crucial factor in drawing up a prevention policy since the accumulation of these displacements over a period of several years can lead to situations of high risk. This work addresses the specific problem related to the prediction of displacements induced by rainfall in unstable areas, of known geology, and crossed by underground pipelines. A neural model has been configured which learns of displacements from instrumented sites (where inclinometric measurements are available) and is able to generalise to other sites not equipped with inclinometers.
\end{abstract}

Key words: unstable areas, pipeline, multilayer perceptron neural network, prediction, multisource data analysis, rainfall

\section{Introduction}

The management of pipelines is a complex and interdisciplinary task, involving the taking of decisions to act based on the evaluation of multiple factors derived from several heterogeneous data sources.

Pipelines are usually distributed over extensive sections of territory and inevitably placed in areas subject to phenomena of geological instability. High-safety monitoring and surveillance must be based on accurate risk assessment, as well as constituting the base for a reliable, optimum management of pipelines. Technological risk assessment, in turn, needs an accurate evaluation of the natural hazard in terms of displacement measures. Private and public companies whose task it is to manage the pipelines normally address this problem by installing adequate instrumentation in the most critical zones. The spreaded structure of the pipelines and the heterogeneity of instrumentation needed, generally make critical conditions to monitor the overall distributed network by direct measurements specific to the area. 
There is then the need to use predictive models capable of reaching generalisations from known measured situations in relation to other pipeline sites not equipped with adequate instrumentation.

Several predictive modelling methodologies are available including physical deterministic approaches (Newmark, 1965; Sarma, 1979; Anderson and Richards 1987; Hoek, 1987) and statistically-based techniques (Davis, 1986) These approaches assume a complete and representative set of data and uniform distribution of multiple data sources. These methods are formally assessed and accurate when these assumptions are true, but they do not provide levels of accuracy and reliability sufficient for many complex applications.

In the context of non standard approaches the interest in neural networks is strongly motivated by their suitability in problems related to multi-source heterogeneous sets of data which do not follow a known statistical distribution (Bishop, 1995). Neural networks are distribution free, and do not require the data to conform to a fixed model, an aspect of great potential in the context of environmental risk studies, which are based on the fusion of multiple, independently acquired sets of data (Benediktsson and Kannellopoulos 1999). Neural networks also prove effective in dealing with noisy contaminated information deriving from imprecision and incompleteness of data.

This work addresses the specific problem associated with the prediction of displacements induced by rainfall in unstable areas, of known geology, crossed by underground pipelines. In pipelines management the accurate prediction of weak displacements is a crucial topic for a prevention policy and the accumulation of these displacements on a multi-year period can determine situations of high risk.

A neural model has been configured which learns displacements from instrumented sites, where inclinometric measurements were available, and is able to generalise to other sites not equipped by inclinometers.

\section{Neural Model}

Neural networks are non-parametric models applied in a variety of fields such as non-linear regression, showing high flexibility in modifying non-linear relationships. Among the variety of network types and architectures, a multi-layer perceptron (MLP) based on back-propagation learning algorithm (Rumelhart et al., 1986) is particularly well suited to dealing with multi-source data.

The basic elements of a network are nodes and links. The nodes are arranged in one input and one output layer, with one or more hidden layers. One hidden layer is usually enough to compute the mapping required by a classification problem (Lipmann, 1987). The output of nodes in one layer is passed to those in the next layer through links that amplify, attenuate or inhibit this output through weighting factors.

The neural network input nodes represent values of multi-source data. Nodes in the output layer are associated with classes in the results of the analysis. In the 
context of the prediction analysis, results are the estimated values of the desired output variable. Each node, above the input level, computes an output related to the weighted sum of its inputs. This can be a final value, or the input for another node. The output of a node $o_{i}$ is calculated by an activation function which usually takes the form of a sigmoid, such as

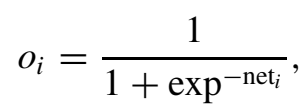

net $_{i}$ is the sum of the weighted inputs to the $i$ th processing node:

$$
\text { net }_{i}=\sum_{j} a_{j} w_{i, j}
$$

where $a_{j}$ is the magnitude of the $j$ th input to the $i$ th node, and $w_{i, j}$ is the weight of the corresponding link.

The weighting factors $w_{i, j}$ constitute the distributed knowledge that the network has to acquire regarding the problem; the weights are determined by means of a learning-by-example algorithm. The generalised delta rule, or back-propagation, is one of the methods most commonly used to adjust the interconnecting weight values in order to achieve a minimal overall training error in a multi-layer network (Rumelhart et al., 1986). As the input patterns are presented during training, the connection weights of the network are adjusted so that the activation of the output nodes matches more closely the desired output patterns. The overall process of training data presentation and weights adjustment is reiterated until the error decreases to an acceptable level, as heuristically assessed. Each iteration is referred to as an epoch. Once training is complete, the network is used in a feed-forward mode to produce prediction results.

The mapping capabilities of a neural network depend upon its structure and other parameters that cannot be set easily and unequivocally on the basis of the characteristics of the problem. In order to construct a network the following aspects must be specified:

- the input/output encoding,

- the number of hidden layers and of nodes in each hidden layer,

- a learning rate controlling the speed of weight change,

- a momentum co-efficient controlling the proportion of weights to be preserved from a previous iteration of network training.

All these parameters are set empirically following general meta-criteria deduced from experience. The network configuration is the result of balanced compromise between opposite empirical criteria. The high variability in neural network configuration, together with the intrinsic critical aspects of over-fitting, "converging to local minima" during learning, make the level of accuracy unpredictable at the start of training. 


\section{Displacement of Unstable Areas Prediction}

Various mechanisms are responsible for initiating mass movements, namely: atmospheric water supply and soil water content; vibration from earthquakes or volcanic eruptions; and land uses involving the artificial increase of slope steepness or application of excessive loads. However, the leading cause of land instability is generally rainfall. Indeed a protracted period of intensive rain leads to high soil saturation thereby promoting conditions propitious to mass movement initiation, development or reactivation (Lopez and Zinck, 1991; CNR, 1994).

Intense and/or prolonged rainfall has a double effect: the kinetic action, that causes direct soil erosion (Ciccacci et al., 1979; Bergsma, 1981; FAO, 1984), and the more complex interaction with the ground water level that is a triggering cause for mass movement.

Besides rainfall measurements acquired from pluviometers placed on slopes affected by instability, a complete characterisation of the kinematic mechanism requires the knowledge of the mass movement geometry, the acquisition of geotechnical parameters, the historical series of displacements. All these data should be consistent, complete and sufficiently representative of the phenomenon in study to make prediction possible.

The reliability level of data available in this application is typical of the practical pipeline management context and is characterised by the following critical aspects:

- geometry data of unstable areas are not updated on a continuous basis

- geotechnical parameters of the material are not available

- rainfall data derive from a grid with a very rough cell resolution

- inclinometers are located along the pipeline and do not always represent the movement, while logs are not acquired with fixed frequency.

Many approaches in the study of slope stability are based on research into establishing a direct relationship between mass movements and rain characteristics in order to define the rainfall threshold values which, once exceeded, may cause mass movements.

Other approaches try to evaluate, through hydrological models, all the parameters of a given rainfall event that interacts in the phenomena such as the amounts of evapo-transpiration, interception, runoff and infiltration, thereby providing information on the levels of soil saturation which are then used to compute slope stability. Such aims are very difficult to reach because of both the complexity of the systems and the need to produce long series of consistent data.

For these reasons, codes based on deterministic method and statistical analysis techniques, to calculate the displacements, do not seem adequate in this context. Under these critical conditions, which are a source of data incompleteness and inconsistency, a predictive model based on a multi-layer perceptron (MLP) is used to generate displacements. 


\subsection{INPUT AND OUTPUT DATA}

The general context of this study is the management of unstable areas crossed by underground pipelines. The data used as input to the network are the geological characteristics of the sites and rainfall. The output results given by the neural model are displacements of the unstable areas. Inclinometer measurements serve to provide known displacement values to train and test the neural model.

\subsubsection{Geological and Geomorphological Data}

Geological and geomorphological information concerning each unstable area came from an existing database containing the following features: geology, lithology, type, stability, evidence and velocity of movement, geometry, morphometry, hydrology, land-use and influence on the infrastructures. The number of areas affected by instability was approximately 150 . These data were characterised by different sources and were therefore not homogeneous: some were numerical, some alphanumerical and others were defined in a heuristic-subjective way.

Table I reports features that were chosen from the information collected in the database, because they were considered to correlate more accurately with the displacements of unstable areas (Luzi and Pergalani, 1996; Belloni et al., 1998; Binaghi et al., 1998; Luzi and Pergalani, 1999).

As a first step, an analysis was performed to establish the range of variation of the selected features and to evaluate the frequency of the geological and geomorphological characteristics. The results of the analysis showed that the most frequent lithology was "clay" (about 200 situations) and the lithologies of deposit were "mainly clay" and "mainly silt" (95 and 90 situations respectively). The most frequent types were translational slide (105 situations) and soil creep (100 situations), and the unstable areas were active (170). The geometric features were: depth of 2-10 m (the peak was 50 cases with $3 \mathrm{~m}$ ), by a length of 100-300 m (60 cases with 100-200 m), and a width of 300-500 m (110 cases with 400-500 m). The slope showed values of between $16 \%$ and $28 \%$, with a peak of $22 \%$ (40 sites) and all the classes of the aspect were present. The water table was assessed 1.5-3 m from the surface (the peak was $2 \mathrm{~m}$ for 75 cases) and no active erosion was present. The most frequently occurring type of land use was farming/pasture and meadow (110 and 85 respectively).

As regard output data, an analysis has been conducted to have a global averaged indication of the range of variation of the displacements and to evaluate how different ranges of displacements are distributed among the areas affected by instability. Results are shown in Figure 1 where the percentages of unstable areas for 5 displacement classes are indicated. 
Table I. Table of the geological and geomorphological features used in the analysis

\begin{tabular}{ll}
\hline Code & Description \\
\hline Site location & \\
Lithology & $\begin{array}{l}\text { Organic clay, Landfill, Gravel, Sand, Sand and gravel, Fine sand, Pyro- } \\
\text { clastic deposit, Silt, Clay, Consolidated clay, Shale, Chaotic clay, Lime- } \\
\text { stone, Sandstone, Marl, Conglomerate, Tuff, Igneous rock, Gneiss }\end{array}$ \\
$\begin{array}{l}\text { Deposit lithology } \\
\text { Landslide type }\end{array}$ & $\begin{array}{l}\text { Mainly clay, Mainly silt, Mainly sand, Mainly gravel, Mixed } \\
\text { Complex, Soil creep }\end{array}$ \\
Landslide stability & Active, Dormant, Inactive \\
Landslide length & $\mathrm{m}$ \\
Landslide width & $\mathrm{m}$ \\
Landslide depth & $\mathrm{m}$ \\
Elevation & $\mathrm{m}$ \\
Slope & $\%$ \\
Aspect & North, North-West, West, South-West, South, South-East, East, North-East \\
Water level & Yes (m), No \\
& Swamp presence Yes, No (if yes, water table 0 m) \\
& Pool presence $\quad$ Yes, No (if yes, water table 0 m) \\
Active erosion & Gully, Rill, Canalised, No active erosion \\
Land use & Cultivated, Vineyard and orchard, Pasture and meadow, Forest, Bare soil, \\
& Re-forested area, Rock or deposit \\
\hline
\end{tabular}

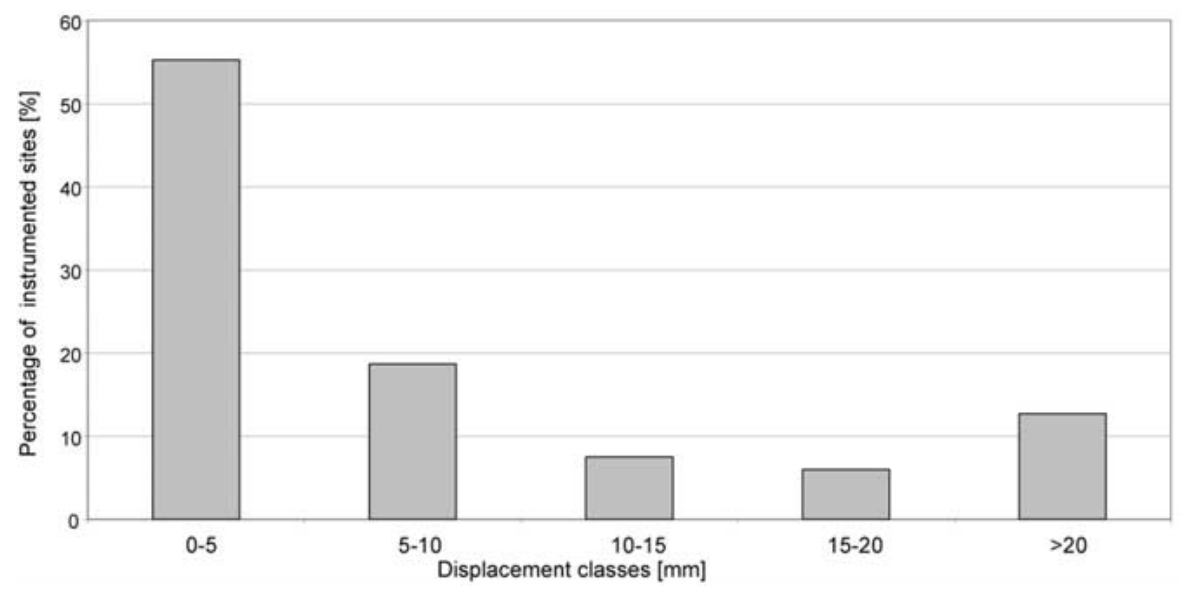

Figure 1. Distribution of instrumented sites for displacements classes. 


\subsubsection{Rainfall Data}

The precipitation data available for the study consisted of weekly rainfall totals, distributed over a grid with a cell measuring $5 \mathrm{~km}$ along the side. These weekly values were derived from daily precipitation measurements recorded at gauge stations randomly distributed over the territory and spatially interpolated to match the grid size. This data set covers a time period of 18 years, starting from the $1980 \mathrm{~s}$, and is a precious source of information for the meteorological characterisation of the sites. Although the time scale is not particularly long, as may occur for hydrological statistics when dealing with meteorological recordings, it does however make it possible to perform an analysis of exceptional precipitation events that may trigger specific movement.

The rainfall data available were pre-elaborated in order to define a series of indexes or rain features, that better describe the dangerous characteristics of the precipitation events within a certain period. These features, with the previously defined geological parameters, provided the input information necessary to instruct the neural network.

In order to characterise the precipitation events that influence displacements, two types of indexes were defined: the first type aims to describe the amount of precipitation between two subsequent inclinometer measurements while the second type tries to outline the influence of the temporal distribution of critical rainfall events within the period.

The features that describe the amount of precipitation are:

- Rl: total amount in the period

$$
R l=\sum_{i=1}^{N} p_{i},
$$

where $p_{i}$ is weekly precipitation value and $N$ is the number of weeks in the period. It represents accumulated rainfall over a meaningful period, i.e., the sum of the weekly values of rain available between two inclinometer measurements, which in our case generally occur every 3 months.

- $R 2$ : total amount related to the seasonal mean rainfall

$$
R 2=\frac{R 1}{\frac{1}{M} \sum_{j=1}^{M} R 1_{j}},
$$

where $M$ is number of years of the data set. It represents the ratio between the cumulated rainfall weekly values and the mean precipitation value, for each grid cell, measured in the same period over the 18 years data set.

- R3: total amount related to the yearly mean rainfall

$$
R 3=\frac{R l}{\frac{1}{M} \sum_{j=1}^{M} \sum_{i=1}^{K} p_{i j}},
$$


where $K$ is the number of weeks (52) in a year. It represents the ratio between the accumulated weekly rainfall values and the mean precipitation value of the year calculated over the 18-year period of the data set (Wiezorek, 1987).

The features that describe the temporal distribution of the critical events are:

- R4: number of critical events

$$
R 4=N_{T},
$$

where $N_{T}$ is the number of weeks in the period with $p_{i}$ values higher a certain threshold $T=\mu_{p}+\sigma$. The term $\mu_{p}$ is the mean value of the yearly maxima for the 18 years data set and $\sigma$ is the standard deviation.

- $R 5 a$ : contribution of critical events

$$
R 5 a=\sum_{i=1}^{N_{T}} p_{i\left(N_{T}\right)} .
$$

This represents the amount of rain carried out by critical events for a period.

- $R 5 b$ : relative contribution of critical events

$$
R 5 b=\frac{R 5 a}{R l} .
$$

The influence of critical events is also described as the ratio with the total amount of rain in a period.

- R6: return period of critical events

$$
R 6=\frac{1}{G},
$$

where $G$ indicates the Gumbel distribution, defined as

$$
G=1+e^{-e^{\frac{p_{i\left(N_{T}\right)}-b}{a}}} \quad \text { with } a=0.78 \sigma \quad b=\mu_{p}-0.5772 a .
$$

This emphasises the outer data as extreme events of the population of the yearly maximum. This return period, in years, is calculated for each maximum of the period with the Gumbel statistic of extreme events (Polemio and Sdao, 1996; Kottegoda and Rosso, 1997).

- R7: seasonal characteristics

This index is expressed by the number of the month in which the weekly data come closest to the inclinometer measurement. It describes the seasonal characterisation of the considered period. 


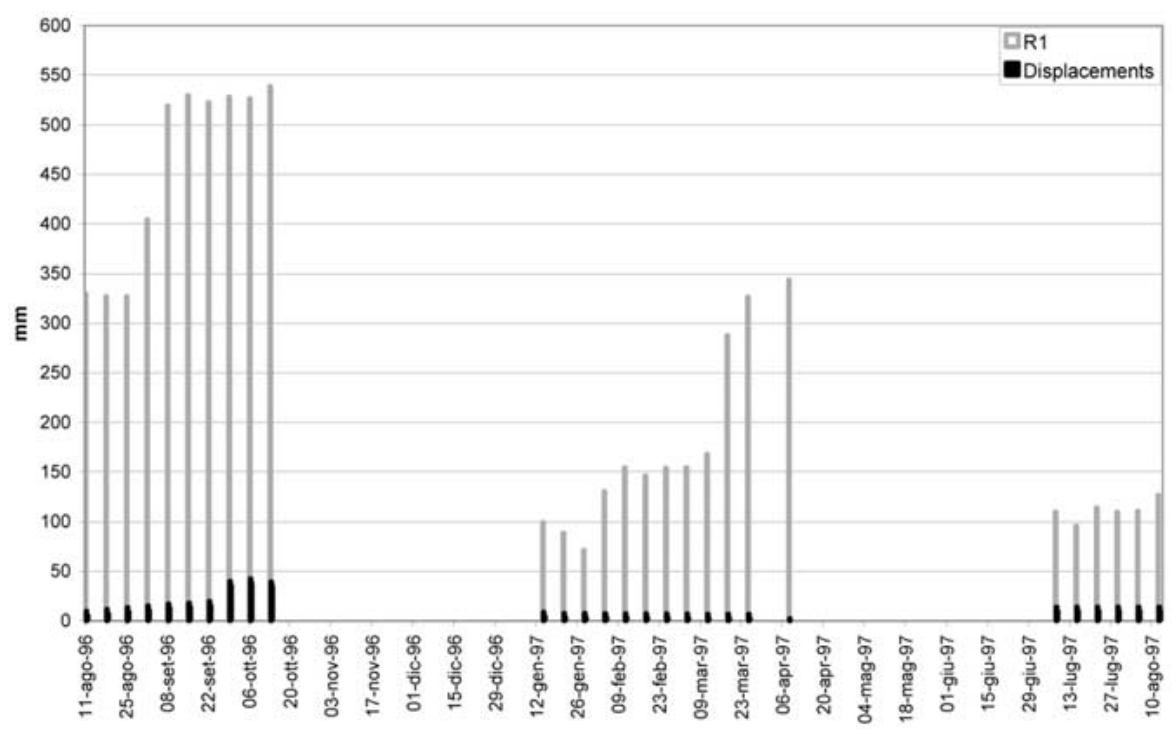

Figure 2. One year data plot, for an example site, showing amplitude of displacement and rainfall $R 1$ feature measurements. Notice the two periods of lack of data.

\subsubsection{Inclinometer Data (Displacements)}

In this application the displacements are provided by processing the inclinometer measurements available in the equipped sites. The displacement here is understood to be the variation recorded by the inclinometers between two consecutive measurements generally occurring every 3 months. In Figure 2 the amplitude of displacements are put in relation with $R l$ feature measurements that represent accumulated rainfall over the 3 months period. In agreement with Wiezorek (1987) and Bozzano et al. (1996), the study proceeds from the idea that the rainfall features selected $(R l, \ldots, R 7)$ can describe the influence of the rainfall events on the mass movement recorded by the instruments.

In each site a series of inclinometers are placed in specific site-dependent positions around the pipeline. Table II details the measuring system, the type and accuracy of the device. Each inclinometer provides measurements at varying depths. As the pipelines may be found at 1 to 3 meters under the ground, only the measurements derived from inclinometers at depths of two meters have been considered in the analysis. A displacement representative of the site is then calculated as the median value among the displacements of all the inclinometers registered at two consecutive measurements. The median value was preferred to the arithmetic mean because it is less sensible to the contaminated measurements represented by the outlines of each series.

As a result of certain omissions in the database of the inclinometers measurements (see Figure 2) it was not possible to compute displacements computed at regular 90-day intervals. To compensate for the incompleteness of the data and 
Table II. Table of the inclinometers characteristics: type and accuracy of the device

\begin{tabular}{ll}
\hline Inclinometer & \\
Sensor & 2 Servoinclinometers \\
Measure range & $\pm 30^{\circ}$ \\
Temperature range & $-5^{\circ} \div 50^{\circ} \mathrm{C}$ \\
Step & $0.5 \mathrm{~m}$ \\
Measure approximation & $\pm 10^{\prime \prime}$ \\
Valuable displacements & $0.05 \mathrm{~mm} / \mathrm{m}$ \\
Linearity & $>0.02 \% \mathrm{~F} . \mathrm{S}$. \\
Sensitivity & $\geq 20000 \sin \alpha(\alpha=$ dip $)$ \\
Automatic readout unit & \\
Display & $\frac{1}{2}$ digits \\
Linearity & $\pm 2 \mathrm{digits}$ \\
Sensitivity & $\geq 20000 \sin \alpha(\alpha=\mathrm{dip})$ \\
Temperature range & $-5^{\circ} \div 50^{\circ} \mathrm{C}$ \\
Cable & \\
Notches & Every $0.5 \mathrm{~m}$ \\
Admissible error & $\pm 5 \mathrm{~cm} / 100 \mathrm{~m}$ \\
Extension by load of $20 \mathrm{~kg}$ & $\leq 0.05 \%$ \\
\hline
\end{tabular}

to obtain an adequate number of displacements to train the neural model, a linear interpolation procedure has been designed to generate intermediate values between two measurements.

\subsection{NETWORK CONFIGURATION}

A series of preliminary experiments was carried out to determine the best configuration for the neural model. Several empirical criteria found in the literature were applied in this phase to limit the range of alternatives and variations to be investigated experimentally (Lipmann, 1987; Paola and Schowengerdt, 1995). The configured network was a simple, fully-connected 3-layer network.

Input data encoding involving the number of neurons assigned for each feature can greatly affect the performance of a neural network. Consequently, three different types of encoding, were tried out: direct, gray, and binary coding. The results obtained confirmed that direct coding, which assigns one neuron to one feature, was the most accurate of the three: the value of each feature data was scaled to the range of $0-1$ and each scaled value presented to a separate input node. The total number of input neurons was 21 , and one output node was used to represent the displacement. The network was designed with one hidden layer. The size of the hidden layer was determined by trial and error, balancing between specialisation 
(more hidden nodes) and generalisation (fewer hidden nodes). The best results were obtained by configuring the hidden layer with 42 neurons according to the empirical rule of dimensioning the hidden layer with twice as many neurons as the number of input neurons (Kanellopoulos and Wilkinson, 1997).

Training a neural network involves establishing a set of learning parameters. An initial parameter concerns the weight updating procedure. According to the canonical form of the gradient descent method (Pao, 1989), batch training should be used to adjust weights after evaluation of the contribution of all the training patterns. In many cases, sequential training is employed, and weight corrections are carried out after each training pattern presentation. In the experiment the last procedure was adopted.

The two inter-dependent parameters Learning Rate and Momentum were determined experimentally, balancing insufficient training against increasing error in the gradient descent process, and set at the beginning of training at the following values: Learning Rate $=0.5 ;$ Momentum $=0.3$.

The training and test phases use a sample data set composed of input-output pairs of data. Input data are described in terms of 11 geological and 8 rainfall features described in previous sections; output data are displacements computed from inclinometers measures as described in previous section. The training set consisted of 2,614 data and the test set of 1,307 data. Of the total of 2,614 training examples only $44(1.7 \%)$ displacements larger than $20 \mathrm{~mm}$ are present.

Figure 3 describes the neural system behaviour: Figure 3a shows the sum of the square errors obtained on training (specialisation) and test (generalisation) data in function of the number of epochs; Figure $3 \mathrm{~b}$ presents the Hinton diagram (1989) of the weights distribution of the hidden and the output layers for 500 and 1,000 epochs. From an absolute point of view, the performance of the neural network increases as the number of epochs increases. However, in this context, the increase of the number of epochs determines an "overspecialisation" in predicting low displacements.

This is supported by Figure 4 where network results after 500 and 1,000 epochs of training are compared with reference test data. The amplitude of predicted displacements become greater than the observed ones with jumping from 500 to 1,000 epochs. This is an expected results in the light of the fact that the number of low amplitude displacements in the training data is considerable higher than the number of high amplitude displacements.

Proceeding from these considerations it was decided to stop the neural learning phase at 500 epochs where the best compromise in predicting both low and high amplitude displacements is verified. Figure 5 exemplifies graphically the final network configuration and its input-output relationships. 
a. Egraph

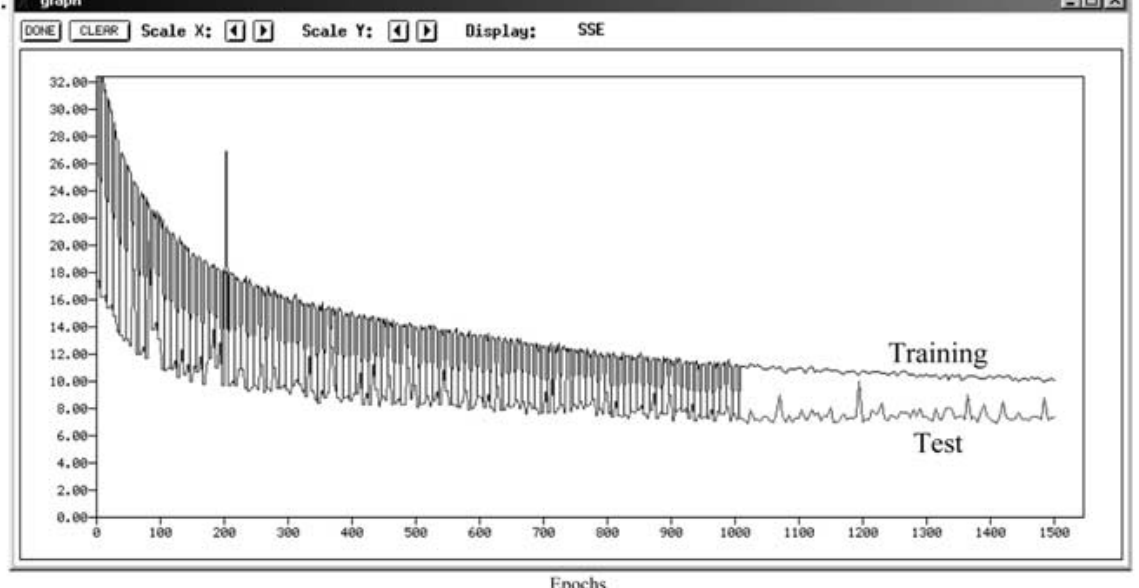

b.

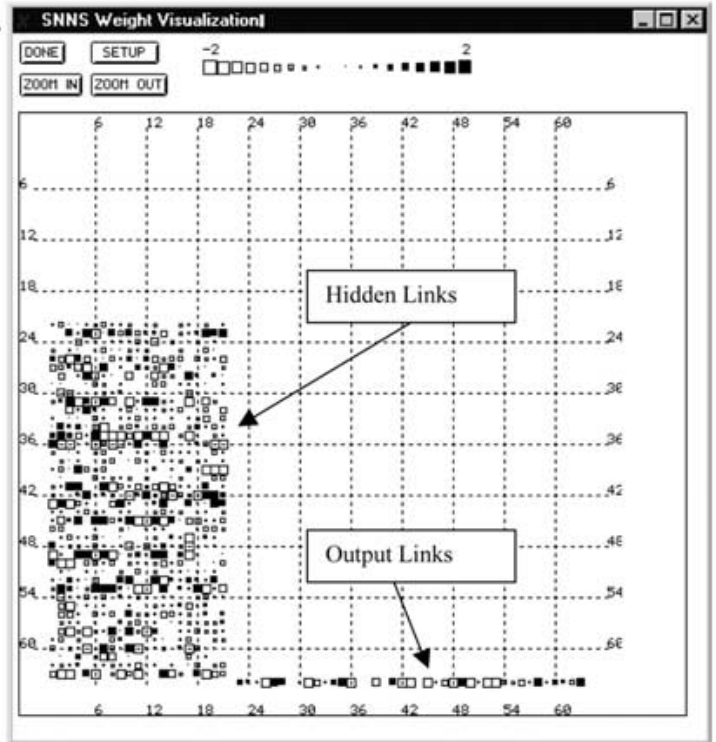

Figure 3. (a) Sum of the square errors (SSE) obtained on training and test data in function of the number of epochs. (b) Hinton diagram of the weight distribution of the hidden and the output layers. The weights are represented as squares (black: positive, white: negative); the size represents the absolute value of the weights.

\subsection{ANALYSIS OF THE RESULTS}

Quantitative evaluation of the result accuracy was conducted with different approaches, in order to complement objective accuracy measurements with the evaluation of the quality of the results in accordance with the project aims. 


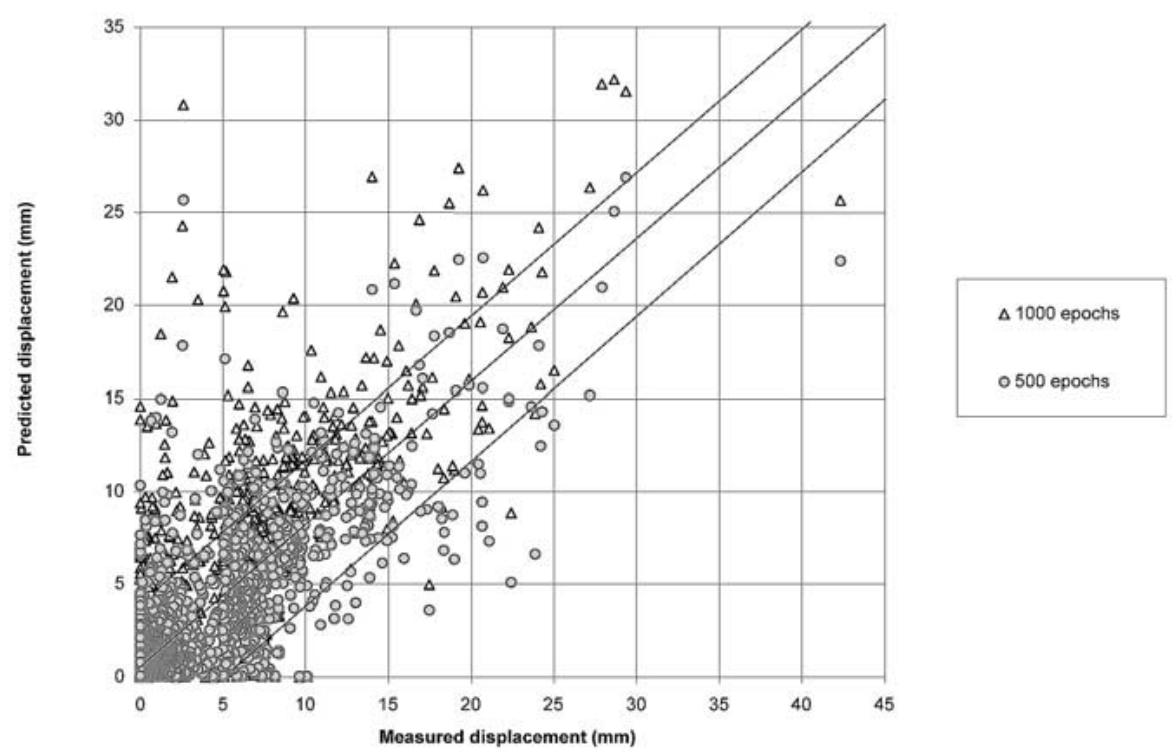

Figure 4. Network results (predicted displacements) after 500 and 1000 epochs of training, compared with reference test data (measured displacements).

The test accuracy level achieved by the final network trained with 500 epochs is $89.2 \%$. This value is computed assuming a coincidence between reference and predicted displacements when their difference lies within the interval $\pm 5 \mathrm{~mm}$. The interval size is set according to inclinometer instrument tolerance.

Figure 6 shows the error magnitude on the basis of displacement values. Most of the errors fall within the interval that defines the instrument tolerance, and the magnitude of the errors becomes significant as the displacement increases. This predictive behaviour of the neural model is due to the fact that large displacement values are scarcely represented in the training data (1.7\%). A detailed analysis of the quality of the results was conducted, comparing the predicted and measured displacements for each specific site over a twelve-month period.

Three representative situations selected from the 150 instrumented sites (Site A, B and C), whose geological and geomorphological characteristics are summarised in Table III, are shown in Figure 7. In Site A and Site B, concerning a typical situation of low and medium displacements, $(<5 \mathrm{~mm}$ and 5-20 $\mathrm{mm})$ clearly predicted values satisfactorily match those measured. In Site C, representative of high displacement $(>20 \mathrm{~mm}$ ), results are less satisfying, showing more relevant differences between predicted and reference data, as globally analysed in Figure 6.

The low quality of these results should be related to the following factors as mentioned earlier in Section 3:

- geological and geomorphological information do not consider the evolution of the mass movement; 


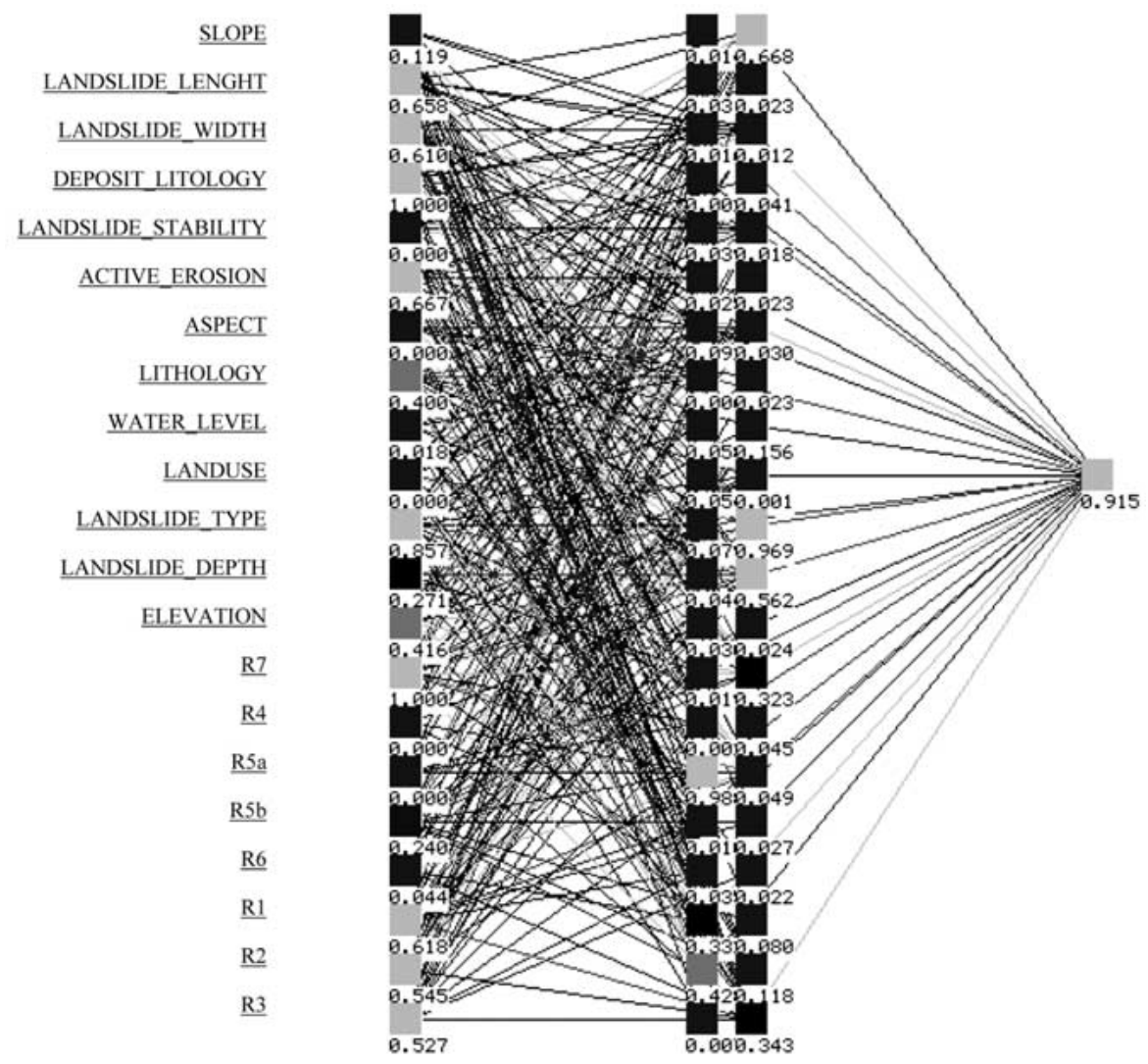

Figure 5. Graphical representation of the network.

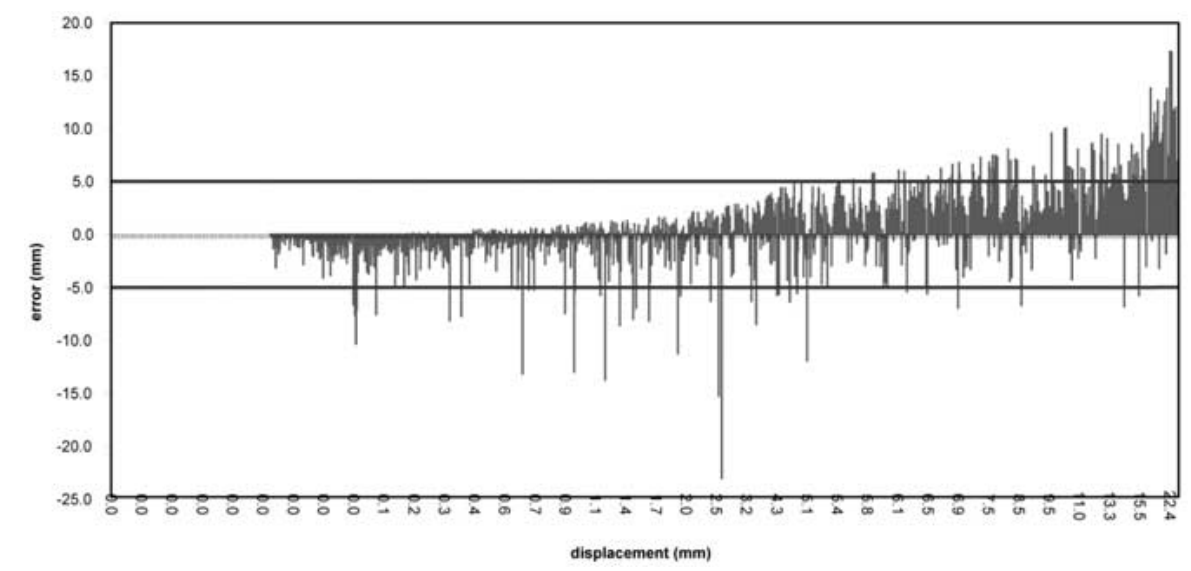

Figure 6. Representation of the error magnitude in function of displacement values. 

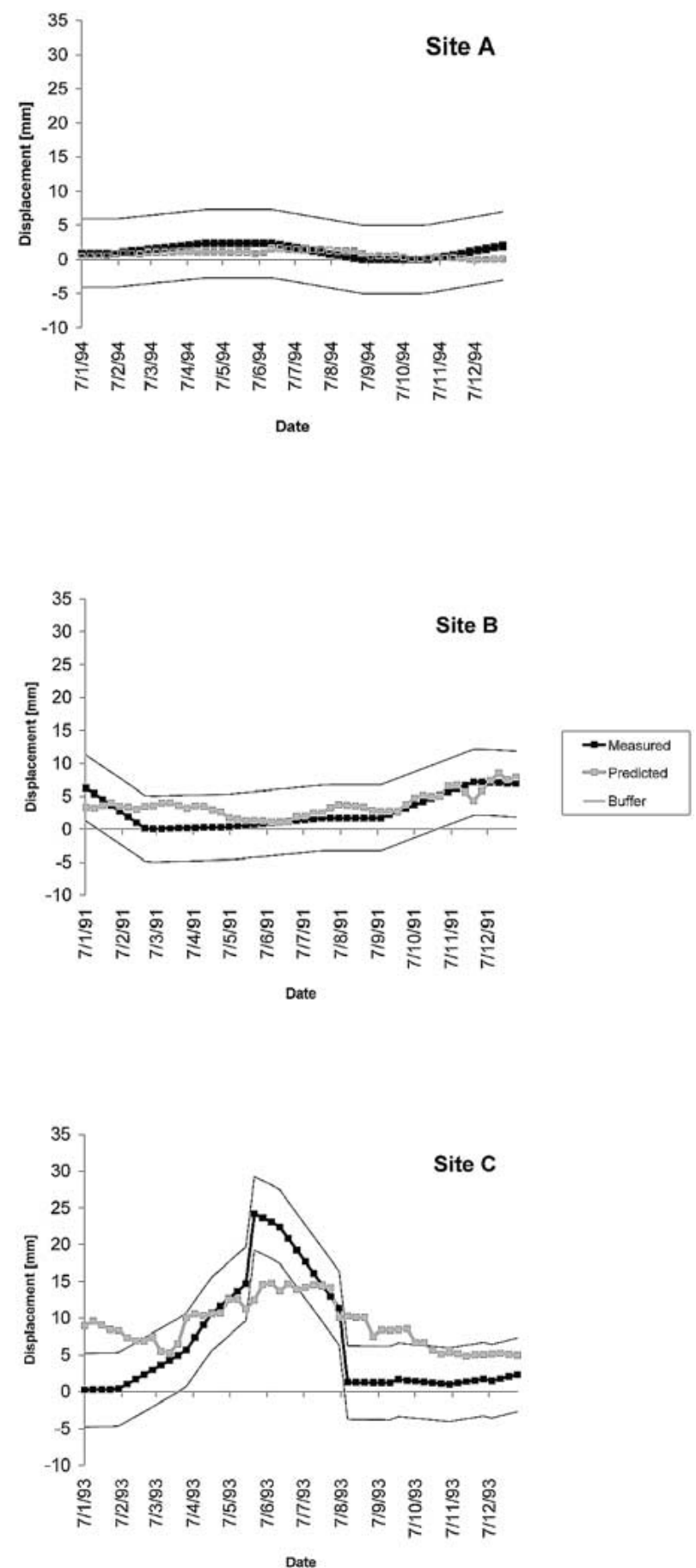

Figure 7. Predicted and measured displacements for a typical situation of low (Site A), medium (Site B) and high (Site C) displacements. 
Table III. Geological and geomorphological characteristics for three representative situations selected from the 200 instrumented sites

\begin{tabular}{llll}
\hline Code & Site A & Site B & Site C \\
\hline Lithology & Clay & Marl & Marl \\
Deposit lithology & Mainly clay & Mainly clay & Mainly clay \\
Landslide type & Translational slide & Translational slide & Translational slide \\
Landslide stability & Active & Active & Active \\
Landslide length & $120 \mathrm{~m}$ & $700 \mathrm{~m}$ & $500 \mathrm{~m}$ \\
Landslide width & $230 \mathrm{~m}$ & $300 \mathrm{~m}$ & $200 \mathrm{~m}$ \\
Landslide depth & $8 \mathrm{~m}$ & $7 \mathrm{~m}$ & $7 \mathrm{~m}$ \\
Elevation & $170 \mathrm{~m}$ & $400 \mathrm{~m}$ & $170 \mathrm{~m}$ \\
Slope & $21 \%$ & $16 \%$ & $32 \%$ \\
Aspect & South-West & North-East & North \\
Water table & $3 \mathrm{~m}$ & $14 \mathrm{~m}$ & $0 \mathrm{~m}$ \\
Active erosion & Gully & Canalised & Canalised \\
Land use & Cultivated & Pasture and meadow & Cultivated \\
\hline
\end{tabular}

- differences between the spatial scale of unstable areas and the grid cell of rainfall data.

These factors induce inconsistencies in the examples used to train the predictive model.

Considering that predicted displacements are used in the context of pipeline management to control mass movements in the sites not equipped by inclinometers, mean annual displacement is the key information required.

Figure 8 shows mean annual displacements computed for the same three sites (Sites A, B and C) already analysed. In the first two sites (Sites A and B) mean annual displacements predicted by the model fit well with the measured values and their differences fall within the tolerance range of inclinometer measurements. Mean annual displacement predicted for Site $\mathrm{C}$ gives a poor approximation of the measured annual trend. However, the approximation is in terms of overestimation allowing a conservative attitude in evaluation and subsequent prevention policy.

To have a global descriptive view of the accuracy of prediction, the maximum differences between predicted and measured mean annual displacements for all cases were computed; results are shown in Figure 9. This type of diagram highlights the worst results of the prediction given by the model: nevertheless only in some cases the differences are relevant, referring to the high displacements. 

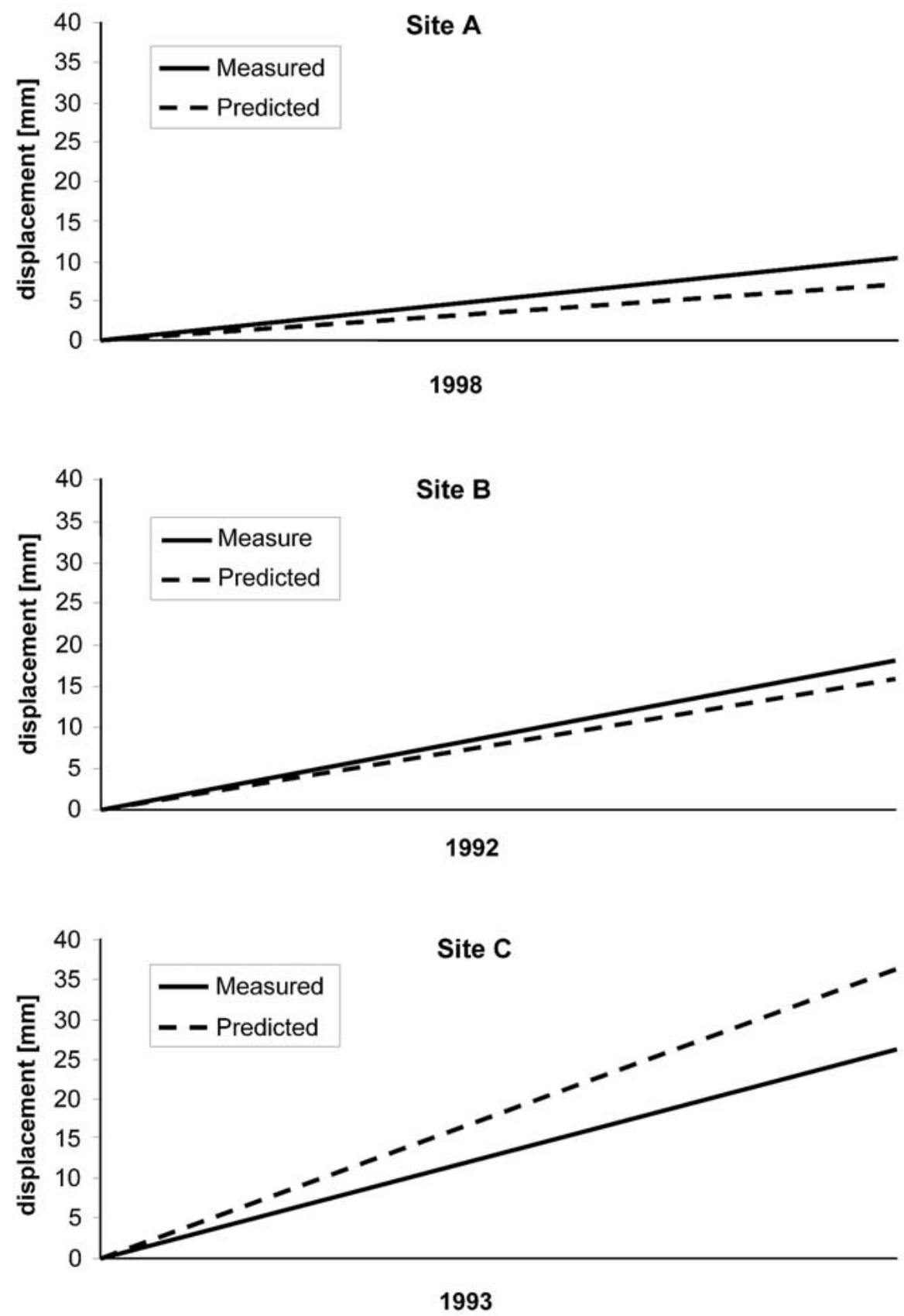

Figure 8. Mean annual displacements computed for Sites A, B, and C. 


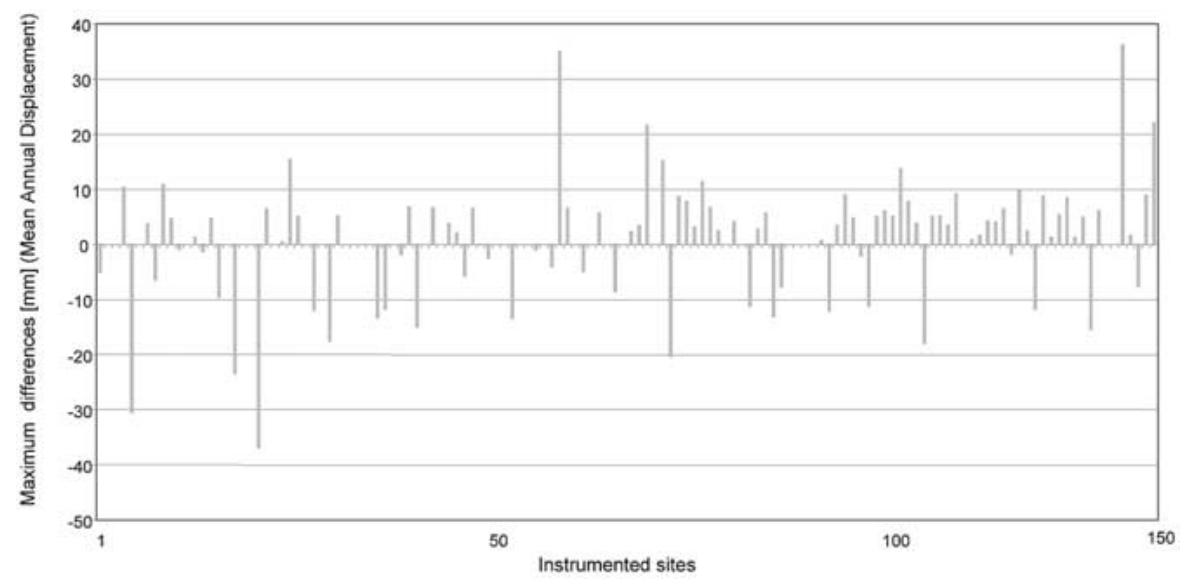

Figure 9. Maximum differences between the predicted and measured mean annual displacements.

\section{Conclusions}

A method for the prediction of displacements induced by rainfall in unstable areas is proposed within the context of pipelines management based on the use of a multilayer perceptron neural network capable of integrating in a unified framework heterogeneous data to produce reliable prediction results. The objective of the study is the accurate prediction of the mean annual displacements whose accumulation on a multi-year period can determine situations of high risk.

Data available in the presented application is typical of practical pipeline management contexts and is characterised by such critical aspects as the lack of certain geomorphological data, the approximate nature of rainfall data, and unstable, outdated area information.

Operating in this critical context, the accuracy of mean annual displacements predicted by the proposed neural model can be considered satisfactory. As seen from the experiments low amplitude displacements are predicted with high accuracy; misleading results obtained for high amplitude displacements go towards conservative estimation.

As both small and large displacements are significant in the cumulative estimation the proposed approach can allow a reliable management of pipelines.

Operationally the use of such a predictive model allows an optimised management of preventative action which would otherwise require the use of a complex network of instrumentation distributed on a large portion of the territory.

\section{References}

Anderson, M. G. and Richards, K. S.: 1987, Slope stability, Geotechnical Engineering and Geomorphology, John Wiley \& Sons, Chichester, pp. 1-648.

Bishop, C. M.: 1995, Neural Network for Pattern Recognition, Clarendon Press, Oxford. 
Belloni, A., Padovan, N., Presbitero, M., Luzi, L., and Pergalani, F.: 1998, Slope instability in static and dynamic conditions addressed to urban planning: The "Oltre Po Pavese" test area Regione Lombardia, Italy, Eleventh European Conference on Earthquake Engineering, Paris, France.

Benediktsson, J. A. and Kannellopoulos, I.: 1999, Information extraction based on multisensor data fusion and neural networks, Information Processing for Remote Sensing, World Scientific Publishing.

Bergsma, E.: 1981, Indices of rain erosivity: A review, ITC Journal 4, 460-484.

Binaghi, E., Luzi, L., Madella, P., Pergalani, F., and Rampini, A.: 1998, Slope instability zonation: A comparison between certainty factor and fuzzy Dempster-Shafer approaches, Natural Hazard 17, 77-97.

Bozzano, F., Floris, M., and Valentini, G.: 1996, Il ruolo delle piogge nelle instabilità di pendio sul versante ionico della Basilicata: Primi risultati, Proc. of the International Conference ALBA 1996, Prevention of Hydrogeological Hazards: The Role of Scientific Research, November 5-7, 1996, Alba, Italy, Vol. I, pp. 357-367.

Ciccacci, S., Fredi, P., and Lupia Palmieri, E.: 1979, Quantitative expression of climatic and geomorphic factors affecting erosional processes: Indirect determination of the amount of erosion in drainage basins in Italy. An approach, Polish-Italian Seminar Superficial Mass Movement in Mountain Regions, May, 1979, Szymbark, Poland.

CNR, G. N. D. C. I.: 1994, Fenomeni Franosi e Centri Abitati, Atti del Convegno Previsione e Prevenzione di Eventi Franosi a Grande Rischio: "Considerazioni sulla valutazione del rischio di frana, 27 Maggio, 1994, Bologna, Italy.

Davis, J. C.: 1986, Statistics and Data Analysis in Geology, John Wiley \& Sons, New York.

FAO: 1984, Methodology Used in the Development of a Soil Loss Rate Map of Ethiopian Highlands, January 1984, Addis Ababa, Ethiopia.

Hinton, G. E: 1989, Connection learning procedures, Artificial Intelligence 40, 185-234.

Hoek E.: 1987, General two-dimensional slope stability analysis, In: E. T. Brown (ed.), Analytical and Computational Method in Engineering Rock Mechanics, London.

Kanellopoulos, I. and Wilkinson, G. G.: 1997, Strategies and best practice for neural network image classification, Int. J. Remote Sensing 18(4), 711-725.

Kottegoda, N. T. and Rosso, R.: 1997, Statistics, Probability, and Reliability for Civil and Environmental Engineers, McGraw Hill, New York.

Lipmann, R. P.: 1987, An introduction to computing with neural nets, IEEE Acoustic, Speech and Signal Processing Magazine 4, 4-22.

Lopez, H. J. and Zinck, J. A.: 1991, GIS-assisted modelling of soil-induced mass movement hazards: A case study of the upper Coello river basin, Tolima, Colombia, ITC Journal 4, 202.

Luzi, L. and Pergalani F.: 1996, A methodology for slope instability zonation using a probabilistic method, 6th Spanish Congress and International Conference on Environmental Geology and Land-Use Planning, Granada, Spain.

Luzi, L. and Pergalani, F.: 1999, Slope instability in static and dynamic conditions for urban planning: The "Oltre Po Pavese" case history (Regione Lombardia-Italy), Natural Hazard 20, 57-82.

Newmark, N. M.: 1965, Effects of earthquakes on dams and embankments, Geotechnique, Vol. 15, London.

Pao, Y. H.: 1989, Adaptive Pattern Recognition and Neural Networks, Addison Wesley, Massachusetts.

Paola, J. D. and Schowengerdt, R. A.: 1995, A review and analysis of back-propagation neural networks for classification of remotely-sensed multi-spectral imagery, International Journal of Remote Sensing 16(16), 3033-3058.

Polemio M. and Sdao, F.: 1996, Landslide hazard and critical rainfall in Southern Italy, VII Int. Symp. on Landslides, Norway, Balkema, Rotterdam, pp. 847-852. 
Rumelhart, H., Hinton, G. E., and Williams, R. J.: 1986, Learning Internal Representation by Error Propagation, In: H. Rumelhart and J. L. McClelland (eds.), Parallel Distributed Processing, MIT Press, Cambridge, MA, pp. 318-362.

Sarma, S. K.: 1979, Stability analysis of embankments and slopes, Journal of the Geotechnical Engineering Division ASCE, 105, No. GT12.

Wiezorek, G. F.: 1987, Effect of rainfall intensity and duration on debris-flow in central Santa Cruz mountains, California. Debris Flow/Avalanches: Process, recognition and mitigation, Reviews in Engineering Geology, Vol. VII, Geological Society of America, Boulder, CO. 